\title{
Interaction of Tertiary Phosphines with Lignin-type, $\alpha, \beta$-Unsaturated Aldehydes in Water
}

\author{
Dmitry V. Moiseev, ${ }^{a}$ Brian O. Patrick, ${ }^{a}$ Brian R. James, ${ }^{a}$ and Thomas Q. Hu ${ }^{b}$ \\ ${ }^{a}$ Department of Chemistry, University of British Columbia, Vancouver, British Columbia, Canada V6T 1Z1 \\ ${ }^{b}$ Pulp and Paper Research Institute of Canada, Vancouver, British Columbia, Canada V6S 2L9
}

\section{Supporting Information}

Table S1. Spectroscopic data for the bis(zwitterionic) compounds $\mathbf{6 b}$ (mixture of $\alpha$ - and $\beta$ atropisomers), and 6c-6g.

Table S2. Spectroscopic and analytical data for the bis(phosphonium) dichlorides 7c-g. 
Table S1. Spectroscopic data for the bis(zwitterionic) compounds $\mathbf{6 b}$ (mixture of $\alpha$ - and $\beta$ atropisomers), and $\mathbf{6 c - 6 g}$

6b (mixture of $\alpha$ - and $\beta$-atropisomers). UV-Vis: 262, $430 \mathrm{~nm}$. Low-resolution ESI MS $\left(\mathrm{H}_{2} \mathrm{O}\right): m / z 457.3(100 \%),\left[6 \mathbf{b}-\mathrm{Et}_{3} \mathrm{P}+\mathrm{H}\right]^{+}$, calcd 457.2.

6b- $\alpha .{ }^{31} \mathrm{P}\left\{{ }^{1} \mathrm{H}\right\}$ NMR: $\delta 44.6\left(\mathrm{~s}, P^{1}\right), 41.8\left(\mathrm{~s}, P^{2}\right) .{ }^{1} \mathrm{H}$ NMR: $\delta 9.30\left(\mathrm{~s}, 1 \mathrm{H}, H^{1}\right), 7.64$ $\left(\mathrm{s}, 1 \mathrm{H}, H^{3}\right), 6.83$ (pseudo d, $\left.{ }^{3} J_{\mathrm{HH}}=7.6,1 \mathrm{H}, H^{12}\right), 6.74\left(H^{13^{\prime}}\right.$ and $H^{8^{\prime}}$ overlapped with $\mathrm{H}^{12}-$ $\beta), 6.43\left(\mathrm{~d},{ }^{3} J_{\mathrm{HH}}=8.4,1 \mathrm{H}, H^{9}\right), 6.37\left(\mathrm{~s}, 1 \mathrm{H}, H^{8}\right), 6.22\left(\mathrm{~s}, 1 \mathrm{H}, H^{12}\right.$, overlapped with $\left.\mathrm{H}^{12}{ }^{\prime}-\beta\right)$, 3.94 [br pseudo t, ${ }^{2} J_{\mathrm{PH}} \approx{ }^{3} J_{\mathrm{HH}} \approx 13,1 \mathrm{H}, H^{4}\left({ }^{1} \mathrm{H}\left\{{ }^{31} \mathrm{P}\right\}: \mathrm{d},{ }^{3} J_{\mathrm{HH}}=11\right)$ ], $3.42\left(\mathrm{~s}, 3 \mathrm{H}, \mathrm{OCH}_{3}{ }^{9}\right)$, 3.30 [br pseudo t, ${ }^{2} J_{\mathrm{PH}} \approx{ }^{3} J_{\mathrm{HH}} \approx 14,1 \mathrm{H}, H^{6}\left({ }^{1} \mathrm{H}\left\{{ }^{31} \mathrm{P}\right\}: \mathrm{d},{ }^{3} J_{\mathrm{HH}}=12\right)$ ], $3.15\left(\mathrm{~s}, 3 \mathrm{H}, \mathrm{OCH}_{3}{ }^{13}\right)$, 3.10 (br m, $1 \mathrm{H}, H^{5}$ ), 2.38 (br m, $1 \mathrm{H}, H^{5}$ ), 2.27- 1.99 (m, $\left.12 \mathrm{H}, \mathrm{P}^{1,2} \mathrm{CH}_{2} \mathrm{CH}_{3}\right), 1.18-0.88(\mathrm{~m}$, $\left.18 \mathrm{H}, \mathrm{P}^{1,2} \mathrm{CH}_{2} \mathrm{CH}_{3}\right) .{ }^{13} \mathrm{C}\left\{{ }^{1} \mathrm{H}\right\}$ NMR: $\delta 197.4\left(\mathrm{~s}, C^{1}\right), 167.1\left(\mathrm{~s}, C^{10}\right), 163.0\left(\mathrm{~d},{ }^{3} J_{\mathrm{PC}}=6.1, C^{3}\right)$, $155.5\left(\mathrm{~s}, C^{14}\right), 151.3\left(\mathrm{~s}, C^{9}\right), 151.0\left(\mathrm{~s}, C^{13}\right), 132.8\left(\mathrm{~s}, C^{8^{\prime}}\right), 119.8\left(\mathrm{~s}, C^{12}\right), 119.2\left(\mathrm{~s}, C^{9^{\prime}}\right)$, $118.9\left(\mathrm{~d},{ }^{2} J_{\mathrm{PC}}=6.1, C^{2}\right), 117.5\left(\mathrm{~s}, C^{7}\right), 115.9\left(\mathrm{~d},{ }^{3} J_{\mathrm{PC}}=4.6, C^{12}\right), 114.3\left(\mathrm{~d},{ }^{2} J_{\mathrm{PC}}=4.6, C^{11}\right)$, $112.4\left(\mathrm{~s}, C^{8}\right), 55.3\left(\mathrm{~s}, \mathrm{OCH}_{3}{ }^{9}\right), 55.1\left(\mathrm{~s}, \mathrm{OCH}_{3}{ }^{13}\right), 36.0\left(\mathrm{dd},{ }^{1} J_{\mathrm{PC}}=45.1,{ }^{3} J_{\mathrm{PC}}=13.0, C^{6}\right)$, $28.8\left(\mathrm{dd},{ }^{1} J_{\mathrm{PC}}=45.9,{ }^{3} J_{\mathrm{PC}}=14.5, C^{4}\right), 24.7\left(\mathrm{~s}, C^{5}\right), 12.0\left(\mathrm{~d},{ }^{1} J_{\mathrm{PC}}=46.7, \mathrm{P}^{1} \mathrm{CH}_{2} \mathrm{CH}_{3}\right), 10.5$ $\left(\mathrm{d},{ }^{1} J_{\mathrm{PC}}=47.4, \mathrm{P}^{2} \mathrm{CH}_{2} \mathrm{CH}_{3}\right), 5.4\left(\mathrm{~d},{ }^{2} J_{\mathrm{PC}}=5.4, \mathrm{P}^{1} \mathrm{CH}_{2} \mathrm{CH}_{3}\right), 4.9\left(\mathrm{~d},{ }^{2} J_{\mathrm{PC}}=5.4, \mathrm{P}^{2} \mathrm{CH}_{2} \mathrm{CH}_{3}\right)$.

6b- $\beta .{ }^{31} \mathrm{P}\left\{{ }^{1} \mathrm{H}\right\}$ NMR: $\delta 44.6\left(\mathrm{~s}, P^{1}\right), 41.8\left(\mathrm{~s}, P^{2}\right) .{ }^{1} \mathrm{H}$ NMR: $\delta 9.30\left(\mathrm{~s}, 1 \mathrm{H}, H^{1}\right), 7.66$ (s, $1 \mathrm{H}, H^{3}$, overlapped with $\left.\mathrm{H}^{3}-\alpha\right), 6.74\left(H^{8^{\prime}}\right.$ and $H^{12}$, overlapped with $\mathrm{H}^{8^{\prime}}-\alpha$ and $\mathrm{H}^{13^{\prime}}-\alpha$ ), $6.43\left(\mathrm{~d},{ }^{3} J_{\mathrm{HH}}=8.4,1 \mathrm{H}, H^{9}\right), 6.36\left(\mathrm{~s}, 1 \mathrm{H}, H^{8}\right), 6.21\left(\mathrm{~d}, 1 \mathrm{H}, H^{13^{\prime}}\right.$ overlapped with $\left.\mathrm{H}^{12}-\alpha\right)$, $6.07\left(\right.$ br d, $\left.{ }^{3} J_{\mathrm{HH}}=6.7,1 \mathrm{H}, H^{12}\right), 3.94$ [br pseudo t, ${ }^{2} J_{\mathrm{PH}} \approx{ }^{3} J_{\mathrm{HH}} \approx 13,1 \mathrm{H}, H^{4}\left({ }^{1} \mathrm{H}\left\{{ }^{31} \mathrm{P}\right\}: \mathrm{d}\right.$, $\left.{ }^{3} J_{\mathrm{HH}}=11\right)$ ], $3.76\left(\mathrm{~s}, 3 \mathrm{H}, \mathrm{OCH}_{3}{ }^{13}\right), 3.42\left(\mathrm{~s}, 3 \mathrm{H}, \mathrm{OCH}_{3}{ }^{9}\right), 3.23$ [br pseudo t, ${ }^{2} J_{\mathrm{PH}} \approx{ }^{3} J_{\mathrm{HH}} \approx 14$, $\left.1 \mathrm{H}, H^{6}\left({ }^{1} \mathrm{H}\left\{{ }^{31} \mathrm{P}\right\}: \mathrm{d},{ }^{3} J_{\mathrm{HH}}=12\right)\right], 3.04\left(\right.$ br m, $\left.1 \mathrm{H}, H^{5}\right), 2.38$ (br m, 1H, $H^{5}$ ), 2.27 to 1.99 (m, $\left.12 \mathrm{H}, \mathrm{P}^{1,2} \mathrm{CH}_{2} \mathrm{CH}_{3}\right), 1.18$ to $0.88\left(\mathrm{~m}, 18 \mathrm{H}, \mathrm{P}^{1,2} \mathrm{CH}_{2} \mathrm{CH}_{3}\right] .{ }^{13} \mathrm{C}\left\{{ }^{1} \mathrm{H}\right\} \mathrm{NMR}: \delta 197.4\left(\mathrm{~s}, C^{1}\right)$, $166.7\left(\mathrm{~s}, C^{10}\right), 163.1\left(\mathrm{~d},{ }^{3} J_{\mathrm{PC}}=6.1, C^{3}\right), 156.5\left(\mathrm{~s}, C^{14}\right), 151.7\left(\mathrm{~s}, C^{13}\right), 151.3\left(\mathrm{~s}, C^{9}\right), 132.1$ $\left(\mathrm{s}, C^{8^{\prime}}\right), 126.8\left(\mathrm{~s}, C^{12^{\prime}}\right), 119.2\left(\mathrm{~s}, C^{9^{\prime}}\right), 119.1$ (presumably d, $C^{2}$, overlapped with $\left.\mathrm{C}^{9^{\prime}}-\alpha, \beta\right)$, $113.2\left(\mathrm{~d},{ }^{2} J_{\mathrm{PC}}=4.6, C^{11}\right), 117.5\left(\mathrm{~s}, C^{7}\right), 112.1\left(\mathrm{~s}, C^{8}\right), 109.6\left(\mathrm{~s}, C^{12}\right), 56.3\left(\mathrm{~s}, \mathrm{OCH}_{3}{ }^{13}\right), 55.3$ $\left(\mathrm{s}, \mathrm{OCH}_{3}{ }^{9}\right), 36.2\left(\mathrm{dd},{ }^{1} J_{\mathrm{PC}}=45.1,{ }^{3} J_{\mathrm{PC}}=13.0, C^{6}\right), 28.6\left(\mathrm{dd},{ }^{1} J_{\mathrm{PC}}=44.4,{ }^{3} J_{\mathrm{PC}}=14.5, C^{4}\right)$, $24.7\left(\mathrm{~s}, C^{5}\right), 12.0\left(\mathrm{~d},{ }^{1} J_{\mathrm{PC}}=46.7, \mathrm{P}^{1} \mathrm{CH}_{2} \mathrm{CH}_{3}\right), 10.4\left(\mathrm{~d},{ }^{1} J_{\mathrm{PC}}=48.2, \mathrm{P}^{2} \mathrm{CH}_{2} \mathrm{CH}_{3}\right), 5.4\left(\mathrm{~d},{ }^{2} J_{\mathrm{PC}}\right.$ $\left.=5.4, \mathrm{P}^{1} \mathrm{CH}_{2} \mathrm{CH}_{3}\right), 4.9\left(\mathrm{~d},{ }^{2} \mathrm{~J}_{\mathrm{PC}}=5.4, \mathrm{P}^{2} \mathrm{CH}_{2} \mathrm{CH}_{3}\right)$. 
6c. ${ }^{31} \mathrm{P}\left\{{ }^{1} \mathrm{H}\right\}$ NMR: $\delta 43.0\left(\mathrm{~d},{ }^{4} J_{\mathrm{PP}}=3, P^{1}\right), 39.5\left(\mathrm{~d},{ }^{4} J_{\mathrm{PP}}=3, P^{2}\right) .{ }^{1} \mathrm{H}$ NMR: $\delta 9.33(\mathrm{~s}$, $\left.1 \mathrm{H}, H^{1}\right), 7.69\left(\mathrm{~s}, 1 \mathrm{H}, H^{3}\right), 6.74\left(\mathrm{~d},{ }^{3} J_{\mathrm{HH}}=8.8,2 \mathrm{H}, H^{8,8^{\prime}}\right), 6.38\left(\mathrm{~d},{ }^{3} J_{\mathrm{HH}}=8.8,2 \mathrm{H}, H^{9,9}\right)$, 7.20-6.00 (br m, 4H, $H^{12,12^{\prime}, 13,13^{\prime}}$ ), 3.94 [br pseudo t, ${ }^{2} J_{\mathrm{PH}} \approx{ }^{3} J_{\mathrm{HH}} \approx 14,1 \mathrm{H}, H^{4}\left({ }^{1} \mathrm{H}\left\{{ }^{31} \mathrm{P}\right\}: \mathrm{d}\right.$, $\left.{ }^{3} J_{\mathrm{HH}}=11\right)$ ], $3.26\left[\right.$ br pseudo $\left.\mathrm{t},{ }^{2} J_{\mathrm{PH}} \approx{ }^{3} J_{\mathrm{HH}} \approx 14,1 \mathrm{H}, H^{6}\left({ }^{1} \mathrm{H}\left\{{ }^{31} \mathrm{P}\right\}: \mathrm{d},{ }^{3} J_{\mathrm{HH}}=12\right)\right], 3.03(\mathrm{br}$ $\left.\mathrm{m}, 1 \mathrm{H}, H^{5}\right), 2.37$ (br m, 1H, $\left.H^{5}\right), 2.28-1.94\left(\mathrm{~m}, 12 \mathrm{H}, \mathrm{P}^{1,2} \mathrm{CH}_{2}\right), 1.18-0.88(\mathrm{~m}, 18 \mathrm{H}$, $\left.\mathrm{P}^{1,2} \mathrm{CH}_{2} \mathrm{C} H_{3}\right) .{ }^{13} \mathrm{C}\left\{{ }^{1} \mathrm{H}\right\}$ NMR $(75 \mathrm{~Hz}): \delta 197.9\left(\mathrm{~s}, \mathrm{C}^{1}\right), 174.5\left(\mathrm{~s}, \mathrm{C}^{10}\right), 167.1\left(\mathrm{~d},{ }^{5} J_{\mathrm{PC}}=1.7\right.$, $\left.C^{14}\right), 162.8\left(\mathrm{~d},{ }^{3} J_{\mathrm{PC}}=6.3, C^{3}\right), 134.8\left(\mathrm{~s}, C^{8,8}\right), 133.7\left(\mathrm{br} \mathrm{s}, C^{12}\right), 127.5\left(\mathrm{br} \mathrm{s}, C^{12}\right), 121.1(\mathrm{~d}$, $\left.{ }^{2} J_{\mathrm{PC}}=5.7, C^{2}\right), 120.3\left(\mathrm{~s}, C^{9,9^{\prime}}\right), 119.9\left(\mathrm{~s}, C^{13,13^{\prime}}\right), 118.2\left(\mathrm{~s}, C^{7}\right), 113.2\left(\mathrm{~d},{ }^{2} J_{\mathrm{PC}}=5.7, C^{11}\right)$, $35.5\left(\mathrm{dd},{ }^{1} J_{\mathrm{PC}}=44.8,{ }^{3} J_{\mathrm{PC}}=13.2, C^{6}\right), 28.4\left(\mathrm{dd},{ }^{1} J_{\mathrm{PC}}=45.4,{ }^{3} J_{\mathrm{PC}}=14.4, C^{4}\right), 24.4\left(\mathrm{~s}, C^{5}\right)$, $11.8\left(\mathrm{~d},{ }^{1} J_{\mathrm{PC}}=46.0, \mathrm{P}^{1} \mathrm{CH}_{2} \mathrm{CH}_{3}\right), 10.4\left(\mathrm{~d},{ }^{1} J_{\mathrm{PC}}=47.1, \mathrm{P}^{2} \mathrm{CH}_{2} \mathrm{CH}_{3}\right), 5.3\left(\mathrm{~d},{ }^{2} J_{\mathrm{PC}}=5.7\right.$, $\left.\mathrm{P}^{1} \mathrm{CH}_{2} \mathrm{CH}_{3}\right), 5.0\left(\mathrm{~d},{ }^{2} J_{\mathrm{PC}}=5.2, \mathrm{P}^{2} \mathrm{CH}_{2} \mathrm{CH}_{3}\right)$. UV-Vis: $254,406 \mathrm{~nm}$. Low-resolution ESI MS (MeOH): $m / z 515.2(11 \%),[6 \mathbf{c}+\mathrm{H}]^{+}$, calcd 515.3; $m / z 397.1(100 \%),\left[6 \mathbf{c}-\mathrm{Et}_{3} \mathrm{P}+\mathrm{H}\right]^{+}$, calcd 397.2.

6d. ${ }^{31} \mathrm{P}\left\{{ }^{1} \mathrm{H}\right\}$ NMR: $\delta 31.9\left(\mathrm{~d},{ }^{4} J_{\mathrm{PP}}=3, P^{1}\right), 30.8\left(\mathrm{~d},{ }^{4} J_{\mathrm{PP}}=3, P^{2}\right) .{ }^{1} \mathrm{H}$ NMR: $\delta 9.21(\mathrm{~s}$, $\left.1 \mathrm{H}, H^{1}\right), 7.57\left(\mathrm{~s}, 1 \mathrm{H}, H^{3}\right), 6.55\left(\mathrm{~s}, 1 \mathrm{H}, H^{12}\right), 6.38\left(\mathrm{~s}, 2 \mathrm{H}, H^{8,8^{\prime}}\right), 6.02\left(\mathrm{~s}, 1 \mathrm{H}, H^{12}\right), 3.83(\mathrm{br}$ pseudo t, ${ }^{2} J_{\mathrm{PH}} \approx{ }^{3} J_{\mathrm{HH}} \approx 13,1 \mathrm{H}, H^{4}$, overlapping with $\mathrm{OCH}_{3}{ }^{13}$ ), 3.79 (s, $3 \mathrm{H}, \mathrm{OCH}_{3}{ }^{13}$ ), 3.54 (s, $6 \mathrm{H}, \mathrm{OCH}_{3}{ }^{9,9}$ ), 3.39 [br pseudo t, ${ }^{2} J_{\mathrm{PH}} \approx{ }^{3} J_{\mathrm{HH}} \approx 14,1 \mathrm{H}, H^{6}\left({ }^{1} \mathrm{H}\left\{{ }^{31} \mathrm{P}\right\}: \mathrm{d},{ }^{3} J_{\mathrm{HH}}=12\right)$ ], 3.21 (s, $3 \mathrm{H}, \mathrm{OCH}_{3}{ }^{13^{\prime}}$ ), 3.11 (br m, $\left.1 \mathrm{H}, H^{5}\right), 2.52\left(\right.$ br m, $\left.1 \mathrm{H}, H^{5}\right), 1.82\left(\mathrm{~d},{ }^{2} J_{\mathrm{PH}}=13.7,9 \mathrm{H}\right.$, $\left.\mathrm{CH}_{3} \mathrm{P}^{1}\right), 1.70\left(\mathrm{~d},{ }^{2} J_{\mathrm{PH}}=13.8,9 \mathrm{H}, \mathrm{CH}_{3} \mathrm{P}^{2}\right) .{ }^{13} \mathrm{C}\left\{{ }^{1} \mathrm{H}\right\}$ NMR: $\delta 196.6\left(\mathrm{~s}, C^{1}\right), 162.2\left(\mathrm{~d},{ }^{3} J_{\mathrm{PC}}=\right.$ 6.1, $\left.C^{3}\right), 158.1\left(\mathrm{~s}, C^{10}\right), 150.8\left(\mathrm{~s}, C^{9,9^{\prime}}\right), 150.6\left(\mathrm{~d},{ }^{4} J_{\mathrm{PC}}=2.3, C^{13}\right), 150.1\left(\mathrm{~s}, C^{13}\right), 143.1(\mathrm{br}$ $\left.\mathrm{s}, C^{14}\right), 119.3\left(\mathrm{~d},{ }^{2} J_{\mathrm{PC}}=6.1, C^{2}\right), 114.8\left(\mathrm{~s}, C^{7}\right), 114.7\left(\mathrm{~d},{ }^{2} J_{\mathrm{PC}}=3.1, C^{11}\right), 111.4\left(\mathrm{br} \mathrm{s}, C^{8,8^{\prime}}\right)$, $111.0\left(\mathrm{~d},{ }^{3} J_{\mathrm{PC}}=4.9, C^{12}\right), 103.3\left(\mathrm{~s}, C^{12}\right), 56.8\left(\mathrm{~s}, \mathrm{OCH}_{3}{ }^{13}\right), 55.9\left(\mathrm{~s}, \mathrm{OCH}_{3}{ }^{9,9}\right), 55.7(\mathrm{~s}$, $\left.\mathrm{OCH}_{3}{ }^{13}\right), 38.8\left(\mathrm{dd},{ }^{1} J_{\mathrm{PC}}=50.8,{ }^{3} J_{\mathrm{PC}}=14.5, C^{6}\right), 32.0\left(\mathrm{dd},{ }^{1} J_{\mathrm{PC}}=52.4,{ }^{3} J_{\mathrm{PC}}=15.3, C^{4}\right), 23.4$ $\left(\mathrm{s}, C^{5}\right), 7.8\left(\mathrm{~d},{ }^{1} J_{\mathrm{PC}}=52.8, \mathrm{P}^{1} \mathrm{CH}_{3}\right), 6.1\left(\mathrm{~d},{ }^{1} J_{\mathrm{PC}}=54.3, \mathrm{P}^{2} \mathrm{CH}_{3}\right)$. UV-Vis: $268,446 \mathrm{~nm}$. Low-resolution ESI MS $\left(\mathrm{H}_{2} \mathrm{O}\right): m / z 475.3(65 \%)$, [6d $\left.\left.-\mathrm{Me}_{3} \mathrm{P}+\mathrm{H}\right]^{+}\right)$, calcd 475.2.

6e. ${ }^{31} \mathrm{P}\left\{{ }^{1} \mathrm{H}\right\}$ NMR: $\delta 40.4\left(\mathrm{~d},{ }^{4} J_{\mathrm{PP}}=3, P^{1}\right), 38.1\left(\mathrm{~d},{ }^{4} J_{\mathrm{PP}}=3, P^{2}\right) .{ }^{1} \mathrm{H}$ NMR $(300$ MHz): $\delta 9.37\left(\mathrm{~s}, 1 \mathrm{H}, H^{1}\right), 7.77\left(\mathrm{~s}, 1 \mathrm{H}, H^{3}\right), 6.65\left(\mathrm{~s}, 1 \mathrm{H}, H^{12}\right), 6.48\left(\mathrm{~s}, 2 \mathrm{H}, H^{8,8^{\prime}}\right), 6.15(\mathrm{~s}, 1 \mathrm{H}$, $\left.H^{12}\right), 4.12$ [br pseudo t, ${ }^{2} J_{\mathrm{PH}} \approx{ }^{3} J_{\mathrm{HH}} \approx 13,1 \mathrm{H}, H^{4}\left({ }^{1} \mathrm{H}\left\{{ }^{31} \mathrm{P}\right\}: \mathrm{d},{ }^{3} J_{\mathrm{HH}}=11\right)$ ], 3.80 (s, $3 \mathrm{H}$, $\left.\mathrm{OCH}_{3}{ }^{13}\right), 3.57\left[\mathrm{t},{ }^{3} J_{\mathrm{HH}}=5.3,12 \mathrm{H}, \mathrm{CH}_{2} \mathrm{OH}\right.$, overlapped with $\mathrm{OCH}_{3}{ }^{9,9}$ ], $3.54(\mathrm{~s}, 6 \mathrm{H}$, $\mathrm{OCH}_{3}{ }^{9,9}$ ), 3.49 [br pseudo t, ${ }^{2} J_{\mathrm{PH}} \approx{ }^{3} J_{\mathrm{HH}} \approx 14,1 \mathrm{H}, H^{6}\left({ }^{1} \mathrm{H}\left\{{ }^{31} \mathrm{P}\right\}: \mathrm{d},{ }^{3} J_{\mathrm{HH}}=13\right)$ ], 3.25 (br m, $1 \mathrm{H}, H^{5}$ ), $3.18\left(\mathrm{~s}, 3 \mathrm{H}, \mathrm{OCH}_{3}{ }^{13^{\prime}}\right.$ ), 2.58 (br m, $1 \mathrm{H}, H^{5}$ ), $2.49-2.14\left(\mathrm{~m}, 12 \mathrm{H}, \mathrm{P}^{1,2} \mathrm{CH}_{2} \mathrm{CH}_{2}\right.$ ), 
1.82-1.35[m, 12H, $\left.\mathrm{P}^{1,2} \mathrm{CH}_{2} \mathrm{CH}\right) \cdot{ }^{13} \mathrm{C}\left\{{ }^{1} \mathrm{H}\right\} \operatorname{NMR}(75 \mathrm{~Hz}): \delta 197.1\left(\mathrm{~s}, \mathrm{C}^{1}\right), 163.2\left(\mathrm{~d},{ }^{3} J_{\mathrm{PC}}=\right.$ 6.3, $\left.C^{3}\right), 158.9\left(\mathrm{~s}, C^{10}\right), 151.6\left(\mathrm{~d},{ }^{4} J_{\mathrm{PC}}=1.7, C^{13}\right), 151.4\left(\mathrm{~s}, C^{13^{\prime}}\right), 150.8\left(\mathrm{~s}, C^{9,9^{\prime}}\right), 147.7(\mathrm{~s}$, $\left.C^{14}\right), 118.3\left(\mathrm{~d},{ }^{2} J_{\mathrm{PC}}=5.7, C^{2}\right), 114.9\left(\mathrm{~s}, C^{7}\right), 111.9\left(\mathrm{br} \mathrm{s}, C^{8,8^{\prime}}\right), 111.4\left(\mathrm{~d},{ }^{3} J_{\mathrm{PC}}=6.3, C^{12^{\prime}}\right)$, $110.9\left(\mathrm{~d},{ }^{2} J_{\mathrm{PC}}=5.7, C^{11}\right), 104.6\left(\mathrm{~d},{ }^{3} J_{\mathrm{PC}}=3.1, C^{12}\right), 61.1\left(\mathrm{~d},{ }^{3} J_{\mathrm{PC}}=16.7, \mathrm{P}^{1} \sim \mathrm{CH}_{2} \mathrm{OH}\right), 60.8$ $\left(\mathrm{d},{ }^{3} \mathrm{~J}_{\mathrm{PC}}=17.2, \mathrm{P}^{2} \sim \mathrm{CH}_{2} \mathrm{OH}\right), 56.9\left(\mathrm{~s}, \mathrm{OCH}_{3}{ }^{13}\right), 56.0\left(\mathrm{~s}, \mathrm{OCH}_{3}{ }^{9,9}\right), 55.6\left(\mathrm{~s}, \mathrm{OCH}_{3}{ }^{13}\right), 37.7$ $\left(\mathrm{dd},{ }^{1} J_{\mathrm{PC}}=45.4,{ }^{3} J_{\mathrm{PC}}=13.2, C^{6}\right), 29.8\left(\mathrm{dd},{ }^{1} J_{\mathrm{PC}}=46.6,{ }^{3} J_{\mathrm{PC}}=14.4, C^{4}\right), 24.6\left(\mathrm{~s}, C^{5}\right), 24.2$ $\left(\mathrm{d},{ }^{2} J_{\mathrm{PC}}=4.0, \mathrm{P}^{1} \mathrm{CH}_{2} \mathrm{CH}_{2}\right), 23.7\left(\mathrm{~d},{ }^{2} J_{\mathrm{PC}}=4.0, \mathrm{P}^{2} \mathrm{CH}_{2} C \mathrm{CH}_{2}\right), 16.0\left(\mathrm{~d},{ }^{1} J_{\mathrm{PC}}=46.6, \mathrm{P}^{1} \mathrm{CH}_{2}\right)$, $14.7\left(\mathrm{~d},{ }^{1} J_{\mathrm{PC}}=47.7, \mathrm{P}^{2} \mathrm{CH}_{2}\right)$. MS (MALDI): $\mathrm{m} / z 815.5(5 \%),[6 \mathbf{e}+\mathrm{H}]^{+}$, calcd 815.4; $\mathrm{m} / z$ $607.5(100 \%),\left[6 e-\mathrm{R}_{3} \mathrm{P}+\mathrm{H}\right]^{+}$, calcd 607.3.

6f. ${ }^{31} \mathrm{P}\left\{{ }^{1} \mathrm{H}\right\}$ NMR: $\delta 29.4\left(\mathrm{~s}, P^{1}\right), 27.4\left(\mathrm{~s}, P^{2}\right) .{ }^{1} \mathrm{H}$ NMR: $\delta 9.19\left(\mathrm{~s}, 1 \mathrm{H}, H^{1}\right), 7.79(\mathrm{t}$, ${ }^{3} J_{\mathrm{HH}}=7.4,1 \mathrm{H}, p-H$ of $\left.\mathrm{P}^{1}\right), 7.68\left(\mathrm{t},{ }^{3} J_{\mathrm{HH}}=7.5,1 \mathrm{H}, p-H\right.$ of $\left.\mathrm{P}^{2}\right), 7.66-7.51(\mathrm{~m}, 8 \mathrm{H}, o-$ and $m$ $H$ of $\left.\mathrm{P}^{1,2}\right), 7.57\left(\mathrm{~s}, 1 \mathrm{H}, H^{3}\right), 6.23\left(\mathrm{~s}, 2 \mathrm{H}, H^{8,8}\right), 5.94\left(\mathrm{~s}, 1 \mathrm{H}, H^{12}\right), 5.39\left(\mathrm{~s}, 1 \mathrm{H}, H^{12}\right), 3.85[\mathrm{br}$ pseudo t, ${ }^{2} J_{\mathrm{PH}} \approx{ }^{3} J_{\mathrm{HH}} \approx 13,1 \mathrm{H}, H^{4}\left({ }^{1} \mathrm{H}\left\{{ }^{31} \mathrm{P}\right\}: \mathrm{d},{ }^{3} J_{\mathrm{HH}}=11\right)$ ], 3.48 [br pseudo $\mathrm{t},{ }^{2} J_{\mathrm{PH}} \approx{ }^{3} J_{\mathrm{HH}} \approx$ $\left.14,1 \mathrm{H}, H^{6}\left({ }^{1} \mathrm{H}\left\{{ }^{31} \mathrm{P}\right\}: \mathrm{d},{ }^{3} J_{\mathrm{HH}}=13\right)\right], 3.41\left(\mathrm{~s}, 6 \mathrm{H}, \mathrm{OCH}_{3}{ }^{9,9}\right), 3.26$ (s, 3H, OCH$\left.{ }_{3}^{13}\right), 3.17$ (br $\left.\mathrm{m}, 1 \mathrm{H}, H^{5}\right), 3.14\left(\mathrm{~s}, 3 \mathrm{H}, \mathrm{OC} H_{3}{ }^{13}\right), 2.30\left(\mathrm{~d},{ }^{2} J_{\mathrm{PH}}=13.9,3 \mathrm{H}, H_{3} \mathrm{CP}^{1}\right), 2.06\left(\mathrm{~d},{ }^{2} J_{\mathrm{PH}}=13.5\right.$, $3 \mathrm{H}, H_{3} \mathrm{CP}^{2}$ ), 2.01 (br m, $\left.1 \mathrm{H}, H^{5^{\prime}}\right), 1.93\left(\mathrm{~d},{ }^{2} J_{\mathrm{PH}}=13.6,6 \mathrm{H}, H_{3} \mathrm{C}^{\prime} \mathrm{P}^{1,2}\right) .{ }^{13} \mathrm{C}\left\{{ }^{1} \mathrm{H}\right\} \mathrm{NMR}: \delta$ $196.5\left(\mathrm{~s}, C^{1}\right), 162.6\left(\mathrm{~d},{ }^{3} J_{\mathrm{PC}}=6.9, C^{3}\right), 158.2\left(\mathrm{~s}, C^{10}\right), 150.7\left(\mathrm{~s}, C^{9,9^{\prime}}\right), 150.3\left(\mathrm{~s}, C^{13,13^{\prime}}\right)$, $144.6\left(\mathrm{~s}, C^{14}\right), 135.0$ (overlapping doublets of $p-C$ of $\left.\mathrm{P}^{1,2}\right), 131.9\left(\mathrm{~d},{ }^{3} J_{\mathrm{PC}}=9.2, m-C\right), 130.6$ $\left(\mathrm{d},{ }^{3} J_{\mathrm{PC}}=9.2, m-C\right), 130.2\left(\mathrm{~d},{ }^{2} J_{\mathrm{PC}}=12.2, o-C\right), 129.8\left(\mathrm{~d},{ }^{2} J_{\mathrm{PC}}=11.5, o-C\right), 119.7\left(\mathrm{~d},{ }^{1} J_{\mathrm{PC}}=\right.$ 81.8, ipso-C-P $\left.\mathrm{P}^{1}\right), 118.7\left(\mathrm{~d},{ }^{2} J_{\mathrm{PC}}=5.4, C^{2}\right), 117.7\left(\mathrm{~d},{ }^{1} J_{\mathrm{PC}}=81.8\right.$, ipso-C- $\left.\mathrm{P}^{2}\right), 114.8\left(\mathrm{~d},{ }^{4} J_{\mathrm{PC}}=\right.$ $\left.1.5, C^{7}\right), 112.6\left(\mathrm{~s}, C^{11}\right), 111.3\left(\mathrm{br} \mathrm{s}, C^{8,8^{\prime}}\right), 111.2\left(\mathrm{~d},{ }^{3} J_{\mathrm{PC}}=5.4, C^{12}\right), 103.1\left(\mathrm{~s}, C^{12}\right), 56.0(\mathrm{~s}$, $\left.\mathrm{OCH}_{3}{ }^{13}\right), 55.8\left(\mathrm{~s}, \mathrm{OCH}_{3}{ }^{8,8^{\prime}}\right.$ and $\left.\mathrm{OCH}_{3}{ }^{13}\right), 40.6\left(\mathrm{dd},{ }^{1} J_{\mathrm{PC}}=48.2,{ }^{3} J_{\mathrm{PC}}=15.3, C^{6}\right), 34.3(\mathrm{dd}$, $\left.{ }^{1} J_{\mathrm{PC}}=50.5,{ }^{3} J_{\mathrm{PC}}=16.1, C^{4}\right), 23.6\left(\mathrm{~s}, C^{5}\right), 5.9\left(\mathrm{~d},{ }^{1} J_{\mathrm{PC}}=52.0, \mathrm{H}_{3} C \mathrm{P}^{1}\right), 5.7\left(\mathrm{~d},{ }^{1} J_{\mathrm{PC}}=55.8\right.$, $\left.\mathrm{H}_{3} C \mathrm{P}^{2}\right), 5.6\left(\mathrm{~d},{ }^{1} J_{\mathrm{PC}}=53.5, \mathrm{H}_{3} C^{\prime} \mathrm{P}^{1}\right), 3.9\left(\mathrm{~d},{ }^{1} J_{\mathrm{PC}}=56.6, \mathrm{H}_{3} C^{\prime} \mathrm{P}^{2}\right) . \mathrm{UV}-\mathrm{Vis}: 260,448 \mathrm{~nm}$. Low-resolution ESI MS $\left(\mathrm{H}_{2} \mathrm{O}\right): m / z 537.2(100 \%),\left[6 \mathbf{f}-\mathrm{Me}_{2} \mathrm{PhP}+\mathrm{H}\right]^{+}$, calcd 537.2; $\mathrm{m} / z$ $139.0(80 \%),\left[\mathrm{Me}_{2} \mathrm{PhP}+\mathrm{H}\right]^{+}$, calcd 139.1.

6g. ${ }^{31} \mathrm{P}\left\{{ }^{1} \mathrm{H}\right\}$ NMR: $\delta 37.4\left(\mathrm{~s}, P^{1}\right), 36.2\left(\mathrm{~s}, P^{2}\right) .{ }^{1} \mathrm{H}$ NMR: $\delta 9.16\left(\mathrm{~s}, 1 \mathrm{H}, H^{1}\right), 6.19(\mathrm{~s}$, $\left.2 \mathrm{H}, H^{8,8^{\prime}}\right), 6.00\left(\mathrm{~s}, 1 \mathrm{H}, H^{12}\right), 5.08\left(\mathrm{~s}, 1 \mathrm{H}, H^{12}\right), 3.77$ [br pseudo t, ${ }^{2} J_{\mathrm{PH}} \approx{ }^{3} J_{\mathrm{HH}} \approx 13,1 \mathrm{H}, H^{4}$ $\left({ }^{1} \mathrm{H}\left\{{ }^{31} \mathrm{P}\right\}:\right.$ br d, $\left.\left.{ }^{3} J_{\mathrm{HH}}=12\right)\right], 3.39$ (br t, $1 \mathrm{H}, H^{6}$ overlapping with $\left.\mathrm{OCH}_{3}{ }^{9,9}\right), 3.41(\mathrm{~s}, 6 \mathrm{H}$, $\mathrm{OCH}_{3}{ }^{9,9}$ ), 3.15 (s, $6 \mathrm{H}, \mathrm{OCH}_{3}{ }^{13,13^{\prime}}$ ), 3.19 (br m, $\left.1 \mathrm{H}, H^{5}\right), 2.81-2.00\left(\mathrm{~m}, 8 \mathrm{H}, \mathrm{P}^{1,2} \mathrm{CH}_{2}\right.$, see text), 1.81 (br m, $1 \mathrm{H}, H^{5}$ ), $1.13-0.90\left(\mathrm{~m}, 12 \mathrm{H}, \mathrm{P}^{1,2} \mathrm{CH}_{2} \mathrm{CH}_{3}\right.$ ). ${ }^{13} \mathrm{C}\left\{{ }^{1} \mathrm{H}\right\}$ NMR: $\delta 197.8$ (s, 
$\left.C^{1}\right), 163.9\left(\mathrm{~d},{ }^{3} J_{\mathrm{PC}}=6.3, C^{3}\right), 159.9\left(\mathrm{~s}, C^{10}\right), 151.9\left(\mathrm{~s}, C^{9,9^{\prime}}\right), 151.0\left(\mathrm{~s}, C^{13^{\prime}}\right), 150.8\left(\mathrm{~s}, C^{13}\right)$, $143.4\left(\mathrm{~s}, C^{14}\right), 136.5\left(\mathrm{~d},{ }^{4} J_{\mathrm{PC}}=3.0, p-C\right.$ of $\left.\mathrm{P}^{1}\right), 136.3\left(\mathrm{~d},{ }^{4} J_{\mathrm{PC}}=3.0, p-C\right.$ of $\left.\mathrm{P}^{2}\right), 134.1(\mathrm{~d}$, ${ }^{3} J_{\mathrm{PC}}=7.9, m-C$ of $\left.\mathrm{P}^{1}\right), 133.0\left(\mathrm{~d},{ }^{3} J_{\mathrm{PC}}=8.1, m-C\right.$ of $\left.\mathrm{P}^{2}\right), 131.5\left(\mathrm{~d},{ }^{2} J_{\mathrm{PC}}=11.8, o-C\right), 131.4$ $\left(\mathrm{d},{ }^{2} J_{\mathrm{PC}}=11.5, o-C\right), 119.3\left(\mathrm{~d},{ }^{2} J_{\mathrm{PC}}=5.6, C^{2}\right), 117.8\left(\mathrm{~d},{ }^{1} J_{\mathrm{PC}}=76.5, i p s o-C-\mathrm{P}^{1}\right), 116.1(\mathrm{~s}$, $\left.C^{7}\right), 115.8\left(\mathrm{~d},{ }^{1} J_{\mathrm{PC}}=76.7\right.$, ipso-C $\left.-\mathrm{P}^{2}\right), 115.4\left(\mathrm{~s}, C^{11}\right), 112.6\left(\mathrm{br} \mathrm{s}, C^{8,8^{\prime}}\right), 111.9\left(\mathrm{~d},{ }^{3} J_{\mathrm{PC}}=5.9\right.$, $\left.C^{12^{\prime}}\right), 104.1\left(\mathrm{~d},{ }^{3} J_{\mathrm{PC}}=2.8, C^{12}\right), 57.1\left(\mathrm{~s}, \mathrm{OCH}_{3}{ }^{9,9^{\prime}}\right), 57.0\left(\mathrm{~s}, \mathrm{OCH}_{3}{ }^{1{ }^{\prime}}\right), 56.9\left(\mathrm{~s}, \mathrm{OCH}_{3}{ }^{13}\right), 38.9$ $\left(\mathrm{dd},{ }^{1} J_{\mathrm{PC}}=44.8,{ }^{3} J_{\mathrm{PC}}=14.2, C^{6}\right), 34.6\left(\mathrm{dd},{ }^{1} J_{\mathrm{PC}}=46.2,{ }^{3} J_{\mathrm{PC}}=14.7, C^{4}\right), 25.7\left(\mathrm{~s}, C^{5}\right), 13.4$ $\left(\mathrm{d},{ }^{1} J_{\mathrm{PC}}=49.0, \mathrm{P}^{1} \mathrm{CH}_{2}{ }^{\prime}\right), 12.4\left(\mathrm{~d},{ }^{1} J_{\mathrm{PC}}=45.9, \mathrm{P}^{1} \mathrm{CH}_{2}\right), 11.6\left(\mathrm{~d},{ }^{1} J_{\mathrm{PC}}=45.9, \mathrm{P}^{2} \mathrm{CH}_{2}\right), 10.5(\mathrm{~d}$, $\left.{ }^{1} J_{\mathrm{PC}}=52.0, \mathrm{P}^{2} \mathrm{CH}_{2}{ }^{\prime}\right), 7.1\left(\mathrm{~d},{ }^{2} J_{\mathrm{PC}}=5.8, \mathrm{P}^{1} \mathrm{CH}_{2} \mathrm{CH}_{3}{ }^{\prime}\right), 6.9\left(\mathrm{~d},{ }^{2} J_{\mathrm{PC}}=5.5, \mathrm{P}^{1} \mathrm{CH}_{2} \mathrm{CH}_{3}\right), 6.2(\mathrm{~d}$, $\left.{ }^{2} J_{\mathrm{PC}}=4.9, \mathrm{P}^{2} \mathrm{CH}_{2} \mathrm{CH}_{3}\right), 5.9\left(\mathrm{~d},{ }^{2} J_{\mathrm{PC}}=4.9, \mathrm{P}^{2} \mathrm{CH}_{2} \mathrm{CH}_{3}\right)$ ). UV-Vis: $268,350,450 \mathrm{~nm}$. Lowresolution ESI MS $\left(\mathrm{H}_{2} \mathrm{O}\right): \mathrm{m} / z 565.3(100 \%)$, [6g $\left.-\mathrm{Et}_{2} \mathrm{PhP}+\mathrm{H}\right]^{+}$, calcd 565.2; $\mathrm{m} / z 167.0$ (53\%), $\left[\mathrm{Et}_{2} \mathrm{PhP}+\mathrm{H}\right]^{+}$, calcd 167.1.

Table S2. Spectroscopic and analytical data for the bis(phosphonium) dichlorides 7c-g

7c. Anal. Calcd for $\mathrm{C}_{30} \mathrm{H}_{46} \mathrm{Cl}_{2} \mathrm{O}_{3} \mathrm{P}_{2}$ : C, 61.33; H, 7.89. Found: C, 61.03; H, 7.87. ${ }^{31} \mathrm{P}\left\{{ }^{1} \mathrm{H}\right\}$ NMR: $\delta 45.0\left(\mathrm{~s}, P^{1}\right), 42.3\left(\mathrm{~s}, P^{2}\right) .{ }^{1} \mathrm{H}$ NMR: $\delta 9.60\left(\mathrm{~s}, 1 \mathrm{H}, H^{1}\right), 7.99\left(\mathrm{~s}, 1 \mathrm{H}, H^{3}\right)$, $7.34\left(\right.$ br s, $\left.1 \mathrm{H}, H^{12}\right), 7.00\left(\right.$ br s, $\left.1 \mathrm{H}, H^{13}\right), 6.93\left(\mathrm{~d},{ }^{3} J_{\mathrm{HH}}=8.7,2 \mathrm{H}, H^{8}\right), 6.81\left(\mathrm{~d},{ }^{3} J_{\mathrm{HH}}=8.7\right.$, $2 \mathrm{H}, H^{9}$ ), 6.70 (br s, $1 \mathrm{H}, H^{12}$ ), 6.41 (br s, $1 \mathrm{H}, H^{13}$ ), 3.92 [br pseudo $\mathrm{t},{ }^{2} J_{\mathrm{PH}} \approx^{3} J_{\mathrm{HH}} \approx 13,1 \mathrm{H}$, $\left.H^{4}\left({ }^{1} \mathrm{H}\left\{{ }^{31} \mathrm{P}\right\}: \mathrm{d},{ }^{3} J_{\mathrm{HH}}=11\right)\right], 3.48\left[\right.$ br pseudo $t,{ }^{2} J_{\mathrm{PH}} \approx{ }^{3} J_{\mathrm{HH}} \approx 13,1 \mathrm{H}, H^{6}\left({ }^{1} \mathrm{H}\left\{{ }^{31} \mathrm{P}\right\}: \mathrm{d},{ }^{3} J_{\mathrm{HH}}=\right.$ 12)], 3.06 (br m, $1 \mathrm{H}, H^{5}$ ), 2.53 (br m, $1 \mathrm{H}, H^{5}$ ), 2.33 to 2.12 (m, 6H, $\mathrm{P}^{1} \mathrm{CH}_{2} \mathrm{CH}_{3}$ ), 2.21 (dq, $\left.6 \mathrm{H},{ }^{2} J_{\mathrm{PH}}=12.5,{ }^{3} J_{\mathrm{HH}}=7.7, \mathrm{P}^{2} \mathrm{CH}_{2} \mathrm{CH}_{3}\right), 1.20\left(\mathrm{dt},{ }^{2} J_{\mathrm{PH}}=18.4,{ }^{3} J_{\mathrm{HH}}=7.7,9 \mathrm{H}, \mathrm{P}^{1} \mathrm{CH}_{2} \mathrm{CH}_{3}\right)$, $1.09\left(\mathrm{dt},{ }^{2} J_{\mathrm{PH}}=18.0,{ }^{3} J_{\mathrm{HH}}=7.7,9 \mathrm{H}, \mathrm{P}^{2} \mathrm{CH}_{2} \mathrm{CH} H_{3}\right) .{ }^{13} \mathrm{C}\left\{{ }^{1} \mathrm{H}\right\}$ NMR: $\delta 200.2\left(\mathrm{~s}, \mathrm{C}^{1}\right), 163.3(\mathrm{~d}$, $\left.{ }^{3} J_{\mathrm{PC}}=7.6, C^{3}\right), 161.0\left(\mathrm{~s}, C^{10}\right), 158.3\left(\mathrm{~s}, C^{14}\right), 135.1\left(\mathrm{br} \mathrm{s}, C^{12}\right), 133.9\left(\mathrm{~s}, C^{8,8^{\prime}}\right), 129.1(\mathrm{br} \mathrm{s}$, $\left.C^{12}\right), 128.3\left(\mathrm{~d},{ }^{2} J_{\mathrm{PC}}=6.1, C^{2}\right), 125.9\left(\mathrm{~d},{ }^{4} J_{\mathrm{PC}}=3.1, C^{7}\right), 120.9\left(\mathrm{~d},{ }^{2} J_{\mathrm{PC}}=6.1, C^{11}\right), 118.2(\mathrm{~s}$, $\left.C^{13,13^{\prime}}\right), 117.6\left(\mathrm{~s}, C^{9,9}\right), 36.5\left(\mathrm{dd},{ }^{1} J_{\mathrm{PC}}=44.4,{ }^{3} J_{\mathrm{PC}}=13.8, C^{6}\right), 29.5\left(\mathrm{dd},{ }^{1} J_{\mathrm{PC}}=45.9,{ }^{3} J_{\mathrm{PC}}=\right.$ 13.8, $\left.C^{4}\right), 25.5\left(\mathrm{~s}, C^{5}\right), 13.3\left(\mathrm{~d},{ }^{1} J_{\mathrm{PC}}=45.9, \mathrm{P}^{1} \mathrm{CH}_{2} \mathrm{CH}_{3}\right), 11.8\left(\mathrm{~d},{ }^{1} J_{\mathrm{PC}}=47.4, \mathrm{P}^{2} \mathrm{CH}_{2} \mathrm{CH}_{3}\right)$, $6.8\left(\mathrm{~d},{ }^{2} J_{\mathrm{PC}}=6.1, \mathrm{P}^{1} \mathrm{CH}_{2} \mathrm{CH}_{3}\right), 6.3\left(\mathrm{~d},{ }^{2} J_{\mathrm{PC}}=6.1, \mathrm{P}^{2} \mathrm{CH}_{2} \mathrm{CH}_{3}\right)$. UV-Vis: $232,340 \mathrm{~nm}$. 
Table S2 (cont).

7d. Anal. Calcd for $\mathrm{C}_{28} \mathrm{H}_{42} \mathrm{Cl}_{2} \mathrm{O}_{7} \mathrm{P}_{2}$ : C, 53.94; H, 6.79. Found: C, 53.60; H, 6.91. ${ }^{31} \mathrm{P}\left\{{ }^{1} \mathrm{H}\right\}$ NMR: $\delta 32.7\left(\mathrm{~s}, P^{1}\right), 31.6\left(\mathrm{~s}, P^{2}\right) .{ }^{1} \mathrm{H}$ NMR: $\delta 9.55\left(\mathrm{~s}, 1 \mathrm{H}, H^{1}\right), 7.90(\mathrm{~d}, J=1.7,1 \mathrm{H}$, $\left.H^{3}\right), 6.58\left(\mathrm{~s}, 1 \mathrm{H}, H^{12}\right), 6.40\left(\mathrm{~s}, 2 \mathrm{H}, H^{8,8^{\prime}}\right), 6.02\left(\mathrm{~s}, 1 \mathrm{H}, H^{12^{\prime}}\right), 3.91$ (br pseudo t, ${ }^{2} J_{\mathrm{PH}} \approx{ }^{3} J_{\mathrm{HH}} \approx$ $\left.12,1 \mathrm{H}, H^{4}\right), 3.77\left(\mathrm{~s}, 3 \mathrm{H}, \mathrm{OCH}_{3}{ }^{13}\right), 3.71\left(\mathrm{~s}, 6 \mathrm{H}, \mathrm{OCH}_{3}{ }^{9,9}\right), 3.57\left[\mathrm{~m}, 1 \mathrm{H}, H^{6}\left({ }^{1} \mathrm{H}\left\{{ }^{31} \mathrm{P}\right\}:\right.\right.$ br d, $\left.\left.{ }^{3} J_{\mathrm{HH}}=13\right)\right], 3.45\left(\mathrm{~s}, 3 \mathrm{H}, \mathrm{OCH}_{3}{ }^{13}\right), 2.91\left(\right.$ br m, $\left.1 \mathrm{H}, H^{5}\right), 2.74\left(\mathrm{br} \mathrm{m}, 1 \mathrm{H}, H^{5}\right), 2.07\left(\mathrm{~d},{ }^{2} J_{\mathrm{PH}}=\right.$ 13.9, 9H, $\left.\mathrm{CH}_{3} \mathrm{P}^{1}\right), 1.78\left(\mathrm{~d},{ }^{2} J_{\mathrm{PH}}=13.9,9 \mathrm{H}, \mathrm{CH}_{3} \mathrm{P}^{2}\right) .{ }^{13} \mathrm{C}\left\{{ }^{1} \mathrm{H}\right\}$ NMR: $\delta 198.2\left(\mathrm{~s}, C^{1}\right), 160.9$ $\left(\mathrm{d},{ }^{3} J_{\mathrm{PC}}=8.4, C^{3}\right), 148.6\left(\mathrm{~d},{ }^{4} J_{\mathrm{PC}}=3.1, C^{13}\right), 148.1\left(\mathrm{~d},{ }^{4} J_{\mathrm{PC}}=3.1, C^{13}\right), 147.5\left(\mathrm{~s}, C^{9,9^{\prime}}\right)$, $138.8\left(\mathrm{~s}, C^{10}\right), 135.0\left(\mathrm{~d},{ }^{5} J_{\mathrm{PC}}=3.8, C^{14}\right), 127.6\left(\mathrm{~d},{ }^{2} J_{\mathrm{PC}}=6.1, C^{2}\right), 123.8\left(\mathrm{~d},{ }^{4} J_{\mathrm{PC}}=1.5, C^{7}\right)$, $119.9\left(\mathrm{~d},{ }^{2} J_{\mathrm{PC}}=6.1, C^{11}\right), 109.3\left(\mathrm{~d},{ }^{3} J_{\mathrm{PC}}=5.4, C^{12}\right), 107.8\left(\mathrm{~s}, C^{8,8^{\prime}}\right), 102.2\left(\mathrm{~d},{ }^{3} J_{\mathrm{PC}}=3.8\right.$, $\left.C^{12}\right), 56.6\left(\mathrm{~s}, \mathrm{OCH}_{3}{ }^{13}\right), 56.0\left(\mathrm{~s}, \mathrm{OCH}_{3}{ }^{9,9}\right), 55.5\left(\mathrm{~s}, \mathrm{OCH}_{3}{ }^{13}\right), 38.4\left(\mathrm{dd},{ }^{1} J_{\mathrm{PC}}=49.7,{ }^{3} J_{\mathrm{PC}}=\right.$ $\left.14.5, C^{6}\right), 31.4\left(\mathrm{dd},{ }^{1} J_{\mathrm{PC}}=53.5,{ }^{3} J_{\mathrm{PC}}=15.3, C^{4}\right), 23.6\left(\mathrm{~d},{ }^{2} J_{\mathrm{PC}}=3.1, C^{5}\right), 8.3\left(\mathrm{~d},{ }^{1} J_{\mathrm{PC}}=53.5\right.$, $\left.\mathrm{P}^{1} \mathrm{CH}_{3}\right), 6.0\left(\mathrm{~d},{ }^{1} J_{\mathrm{PC}}=53.5, \mathrm{P}^{2} \mathrm{CH}_{3}\right)$. UV-Vis: $248,362 \mathrm{~nm}$. Low-resolution ESI MS $(\mathrm{MeOH}): m / z 587.2(100 \%),[7 \mathbf{d}-\mathrm{Cl}]^{+}$, calcd 587.2; $\mathrm{m} / z 475.2(54 \%),\left[7 \mathbf{d}-2 \mathrm{Cl}-\mathrm{Me}_{3} \mathrm{P}-\right.$ $\mathrm{H}]^{+}$, calcd 475.2; $m / 2 z 276.2(20 \%),[7 d-2 \mathrm{Cl}]^{2+}$, calcd 276.6 .

7e. ${ }^{31} \mathrm{P}\left\{{ }^{1} \mathrm{H}\right\}$ NMR: $\delta 40.8\left(\mathrm{~s}, P^{1}\right), 38.3\left(\mathrm{~s}, P^{2}\right) .{ }^{1} \mathrm{H}$ NMR: $\delta 9.57\left(\mathrm{~s}, 1 \mathrm{H}, H^{1}\right), 7.90(\mathrm{~s}$, $\left.1 \mathrm{H}, H^{3}\right), 6.69\left(\mathrm{~s}, 1 \mathrm{H}, H^{12}\right), 6.36\left(\mathrm{~s}, 2 \mathrm{H}, H^{8,8^{\prime}}\right), 6.20\left(\mathrm{~s}, 1 \mathrm{H}, H^{12^{\prime}}\right), 3.95$ [br pseudo t, ${ }^{2} J_{\mathrm{PH}} \approx$ $\left.{ }^{3} J_{\mathrm{HH}} \approx 13,1 \mathrm{H}, H^{4}\left({ }^{1} \mathrm{H}\left\{{ }^{31} \mathrm{P}\right\}: \mathrm{d},{ }^{3} J_{\mathrm{HH}}=11\right)\right], 3.85\left(\mathrm{~s}, 3 \mathrm{H}, \mathrm{OCH}_{3}{ }^{13}\right), 3.63-3.50(\mathrm{~m}, 12 \mathrm{H}$, $\mathrm{CH}_{2} \mathrm{OH}$ ), 3.64 (s, 6H, OCH${ }_{3}{ }^{9,9^{\prime}}$ ), (br pseudo t, $\mathrm{H}^{6}$ overlapping with $\mathrm{CH}_{2} \mathrm{OH}$ ), 3.07 (br m, $\left.1 \mathrm{H}, H^{5}\right), 3.35$ (s, 3H, $\mathrm{OCH}_{3}{ }^{13}$ ), 2.77 (br m, $1 \mathrm{H}, H^{5^{\prime}}$ ), 2.59-2.20 (m, 12H, $\mathrm{P}^{1,2} \mathrm{CH}_{2} \mathrm{CH}_{2}$ ), 1.82-1.38 (m, 12H, $\left.\mathrm{P}^{1,2} \mathrm{CH}_{2} \mathrm{CH}_{2}\right) .{ }^{13} \mathrm{C}\left\{{ }^{1} \mathrm{H}\right\} \mathrm{NMR}: \delta 200.2\left(\mathrm{~s}, C^{1}\right), 163.5\left(\mathrm{~d},{ }^{3} J_{\mathrm{PC}}=7.6, C^{3}\right)$, $150.2\left(\mathrm{~d},{ }^{4} J_{\mathrm{PC}}=3.1, C^{13}\right), 149.7\left(\mathrm{~d},{ }^{4} J_{\mathrm{PC}}=2.3, C^{13^{\prime}}\right), 149.0\left(\mathrm{~s}, C^{9,9^{\prime}}\right), 140.9\left(\mathrm{~s}, C^{10}\right), 136.9$ $\left(\mathrm{d},{ }^{5} J_{\mathrm{PC}}=7.6, C^{14}\right), 127.4\left(\mathrm{~d},{ }^{2} J_{\mathrm{PC}}=6.1, C^{2}\right), 125.0\left(\mathrm{~s}, C^{7}\right), 120.8\left(\mathrm{~d},{ }^{2} J_{\mathrm{PC}}=6.1, C^{11}\right), 110.9$ $\left(\mathrm{d},{ }^{3} J_{\mathrm{PC}}=6.9, C^{12}\right), 110.1\left(\mathrm{~s}, C^{8,8^{\prime}}\right), 104.2\left(\mathrm{~s}, C^{12}\right), 62.3\left(\left[\mathrm{~d},{ }^{3} J_{\mathrm{PC}}=16.8, \mathrm{P}^{1} \sim C \mathrm{H}_{2} \mathrm{OH}\right), 62.0\right.$ $\left(\mathrm{d},{ }^{3} J_{\mathrm{PC}}=16.8, \mathrm{P}^{2} \sim \mathrm{CH}_{2} \mathrm{OH}\right), 58.1\left(\mathrm{~s}, \mathrm{OCH}_{3}{ }^{13}\right), 57.5\left(\mathrm{~s}, \mathrm{OCH}_{3}{ }^{9,9}\right), 56.9\left(\mathrm{~s}, \mathrm{OCH}_{3}{ }^{13}\right), 38.4$ $\left(\mathrm{dd},{ }^{1} J_{\mathrm{PC}}=44.8,{ }^{3} J_{\mathrm{PC}}=13.4, C^{6}\right), 31.1\left(\mathrm{dd},{ }^{1} J_{\mathrm{PC}}=46.7,{ }^{3} J_{\mathrm{PC}}=15.3, C^{4}\right), 25.3\left(\mathrm{~s}, C^{5}\right), 25.7$ $\left(\mathrm{d},{ }^{2} J_{\mathrm{PC}}=3.8, \mathrm{P}^{1} \mathrm{CH}_{2} \mathrm{CH}_{2}\right), 25.1\left(\mathrm{~d},{ }^{2} J_{\mathrm{PC}}=3.8, \mathrm{P}^{2} \mathrm{CH}_{2} \mathrm{CH}_{2}\right), 17.4\left(\mathrm{~d},{ }^{1} J_{\mathrm{PC}}=46.7, \mathrm{P}^{1} \mathrm{CH}_{2}\right)$, $15.9\left(\mathrm{~d},{ }^{1} J_{\mathrm{PC}}=47.4, \mathrm{P}^{2} \mathrm{CH}_{2}\right)$. Low-resolution ESI MS (MeOH): $m / 2 z 408.2(35 \%)$, [7e $2 \mathrm{Cl}]^{2+}$, calcd 408.7; m/z $607.2(42 \%),\left[7 \mathrm{e}-2 \mathrm{Cl}-\left(\mathrm{HOCH}_{2} \mathrm{CH}_{2} \mathrm{CH}_{2}\right)_{3} \mathrm{P}-\mathrm{H}\right]^{+}$, calcd 607.3; $m / z 851.3(15 \%),[7 \mathbf{e}-\mathrm{Cl}]^{+}$, calcd 851.4; m/z $209.1(100 \%),\left[\left\{\left(\mathrm{CH}_{2}\right)_{3} \mathrm{OH}\right\}_{3} \mathrm{P}+\mathrm{H}\right]^{+}$, calcd 209.1). 
Table S2 (cont).

7f. ${ }^{31} \mathrm{P}\left\{{ }^{1} \mathrm{H}\right\}$ NMR: $\delta 30.1\left(\mathrm{~s}, P^{1}\right), 28.7\left(\mathrm{~s}, P^{2}\right) .{ }^{1} \mathrm{H}$ NMR: $\delta 9.52\left(\mathrm{~s}, 1 \mathrm{H}, H^{1}\right)$, 7.89-7.55 (m, 10H, $\mathrm{C}_{6} H_{5}$ of $\left.\mathrm{P}^{1,2}\right), 7.84\left(\mathrm{~s}, 1 \mathrm{H}, H^{3}\right), 6.21\left(\mathrm{~s}, 2 \mathrm{H}, H^{8,8^{\prime}}\right), 5.93\left(\mathrm{~s}, 1 \mathrm{H}, H^{12^{\prime}}\right)$, $5.40\left(\mathrm{~s}, 1 \mathrm{H}, H^{12}\right), 3.85\left[\right.$ br pseudo $\left.\mathrm{t},{ }^{2} J_{\mathrm{PH}} \approx{ }^{3} J_{\mathrm{HH}} \approx 13,1 \mathrm{H}, H^{4}\left({ }^{1} \mathrm{H}\left\{{ }^{31} \mathrm{P}\right\}: \mathrm{d},{ }^{3} J_{\mathrm{HH}}=12\right)\right], 3.65$ [br pseudo t, $\left.{ }^{2} J_{\mathrm{PH}} \approx{ }^{3} J_{\mathrm{HH}} \approx 14,1 \mathrm{H}, H^{6}\left({ }^{1} \mathrm{H}\left\{{ }^{31} \mathrm{P}\right\}: \mathrm{d},{ }^{3} J_{\mathrm{HH}}=13\right)\right], 3.57\left(\mathrm{~s}, 6 \mathrm{H}, \mathrm{OCH}_{3}{ }^{9,9}\right), 3.33$ $\left(\mathrm{s}, 3 \mathrm{H}, \mathrm{OCH}_{3}{ }^{13}\right), 3.30\left(\mathrm{~s}, 3 \mathrm{H}, \mathrm{OCH}_{3}{ }^{13}\right), 3.12\left(\mathrm{br} \mathrm{m}, 1 \mathrm{H}, H^{5}\right), 2.50\left(\mathrm{~d},{ }^{2} \mathrm{~J}_{\mathrm{PH}}=13.9,3 \mathrm{H}\right.$, $H_{3} \mathrm{CP}^{1}$ ), 2.23 (br m, $1 \mathrm{H}, H^{5}$, overlapping with $\left.H_{3} \mathrm{CP}^{2}\right), 2.22\left(\mathrm{~d},{ }^{2} J_{\mathrm{PH}}=13.6,3 \mathrm{H}, H_{3} \mathrm{CP}^{2}\right.$ ), $2.20\left(\mathrm{~d},{ }^{2} J_{\mathrm{PH}}=13.7,3 \mathrm{H}, H_{3} \mathrm{C}^{\prime} \mathrm{P}^{1}\right), 2.08\left(\mathrm{~d},{ }^{2} J_{\mathrm{PH}}=13.6,3 \mathrm{H}, H_{3} \mathrm{C}^{\prime} \mathrm{P}^{2}\right) .{ }^{13} \mathrm{C}\left\{{ }^{1} \mathrm{H}\right\} \mathrm{NMR}: \delta$ $198.2\left(\mathrm{~s}, C^{1}\right), 161.7\left(\mathrm{~d},{ }^{3} J_{\mathrm{PC}}=7.6, C^{3}\right), 148.0\left(\mathrm{~d},{ }^{4} J_{\mathrm{PC}}=2.3, C^{13}\right), 147.9\left(\mathrm{~d},{ }^{4} J_{\mathrm{PC}}=3.1, C^{13}\right)$, $147.4\left(\mathrm{~s}, C^{9,9^{\prime}}\right), 139.0\left(\mathrm{~s}, C^{10}\right), 135.4\left(\mathrm{~d},{ }^{4} J_{\mathrm{PC}}=3.1, p-C\right), 135.3\left(\mathrm{~d},{ }^{4} J_{\mathrm{PC}}=3.1, p-C\right), 135.1$ $\left(\mathrm{d},{ }^{3} J_{\mathrm{PC}}=3.8, C^{14}\right), 132.0\left(\mathrm{~d},{ }^{3} J_{\mathrm{PC}}=9.9, m-C\right), 130.7\left(\mathrm{~d},{ }^{3} J_{\mathrm{PC}}=9.9, m-C\right), 130.4\left(\mathrm{~d},{ }^{2} J_{\mathrm{PC}}=\right.$ $12.2, o-C), 129.9\left(\mathrm{~d},{ }^{2} J_{\mathrm{PC}}=12.2, o-C\right), 126.7\left(\mathrm{~d},{ }^{2} J_{\mathrm{PC}}=6.1, C^{2}\right), 123.8\left(\mathrm{~d},{ }^{4} J_{\mathrm{PC}}=2.3, C^{7}\right)$, $\left.119.8\left[\mathrm{~d},{ }^{1} J_{\mathrm{PC}}=82.6, C_{\mathrm{ipso}}-\mathrm{P}^{1}\right), 119.1\left(\mathrm{~d},{ }^{2} J_{\mathrm{PC}}=6.1, C^{11}\right), 117.1 \mathrm{~d},{ }^{1} J_{\mathrm{PC}}=84.1, C_{\mathrm{ipso}}-\mathrm{P}^{2}\right)$, $109.4\left(\mathrm{~d},{ }^{3} J_{\mathrm{PC}}=6.1, C^{12}\right), 108.0\left(\mathrm{~s}, C^{8,8^{\prime}}\right), 102.0\left(\mathrm{~d},{ }^{3} J_{\mathrm{PC}}=4.6, C^{12}\right), 56.1\left(\mathrm{~s}, \mathrm{OCH}_{3}{ }^{8,8^{\prime}}\right), 56.0$ $\left(\mathrm{s}, \mathrm{OCH}_{3}{ }^{12}\right), 55.5\left(\mathrm{~s}, \mathrm{OCH}_{3}{ }^{12}\right), 40.1\left(\mathrm{dd},{ }^{1} J_{\mathrm{PC}}=47.4,{ }^{3} J_{\mathrm{PC}}=13.8, C^{6}\right), 34.0\left(\mathrm{dd},{ }^{1} J_{\mathrm{PC}}=51.2\right.$, $\left.{ }^{3} J_{\mathrm{PC}}=14.6, C^{4}\right), 23.7\left(\mathrm{~s}, C^{5}\right), 6.3\left(\mathrm{~d},{ }^{1} J_{\mathrm{PC}}=53.5, \mathrm{H}_{3} C \mathrm{P}^{1}\right), 5.7\left(\mathrm{~d},{ }^{1} J_{\mathrm{PC}}=55.1, \mathrm{H}_{3} C^{\prime} \mathrm{P}^{1}\right), 5.0$ $\left(\mathrm{d},{ }^{1} J_{\mathrm{PC}}=52.8, \mathrm{H}_{3} C \mathrm{P}^{2}\right), 4.2\left(\mathrm{~d},{ }^{1} J_{\mathrm{PC}}=56.6, \mathrm{H}_{3} C^{\prime} \mathrm{P}^{2}\right)$. UV-Vis: $248,368 \mathrm{~nm}$. Low-resolution ESI MS (MeOH): $m / z 537.4(100 \%),\left[7 f-2 \mathrm{Cl}-\mathrm{Me}_{2} \mathrm{PhP}-\mathrm{H}\right]^{+}$, calcd 537.2; $[\mathrm{m} / z 711.4$ $(22 \%),[7 f-C l]^{+}$, calcd 711.2; $m / z 139.0(30 \%),\left[\mathrm{Me}_{2} \mathrm{PhP}+\mathrm{H}\right]^{+}$, calcd 139.1.

7g. ${ }^{31} \mathrm{P}\left\{{ }^{1} \mathrm{H}\right\}$ NMR: $\delta 37.9\left(\mathrm{~s}, P^{1}\right), 37.0\left(\mathrm{~s}, P^{2}\right) .{ }^{1} \mathrm{H}$ NMR: $\delta 9.52\left(\mathrm{~s}, 1 \mathrm{H}, H^{1}\right), 7.88-$ $7.50\left(\mathrm{~m}, 10 \mathrm{H}, \mathrm{C}_{6} H_{5}\right), 7.80\left(\mathrm{~s}, 1 \mathrm{H}, H^{3}\right), 6.17\left(\mathrm{~s}, 2 \mathrm{H}, H^{8,8^{\prime}}\right), 6.12\left(\mathrm{~s}, 1 \mathrm{H}, H^{12^{\prime}}\right), 5.17(\mathrm{~s}, 1 \mathrm{H}$, $H^{12}$ ), 3.79 [br pseudo $\mathrm{t},{ }^{2} J_{\mathrm{PH}} \approx{ }^{3} J_{\mathrm{HH}} \approx 13,1 \mathrm{H}, H^{4}\left({ }^{1} \mathrm{H}\left\{{ }^{31} \mathrm{P}\right\}:\right.$ br d, $\left.{ }^{3} J_{\mathrm{HH}}=12\right)$ ], $3.58(\mathrm{~s}, 6 \mathrm{H}$, $\left.\mathrm{OCH}_{3}{ }^{9,9^{\prime}}\right), 3.57\left(1 \mathrm{H}, \mathrm{H}^{6}\right.$ overlapping with $\left.\mathrm{OCH}_{3}{ }^{9,9^{\prime}}\right), 3.38\left(\mathrm{~s}, 3 \mathrm{H}, \mathrm{OCH}_{3}{ }^{13^{\prime}}\right), 3.22(\mathrm{~s}, 3 \mathrm{H}$, $\mathrm{OCH}_{3}{ }^{13}$ ), 3.14 (br m, $1 \mathrm{H}, H^{5}$ ), 3.08-2.25 (m, 8H, $\mathrm{P}^{1,2} \mathrm{CH}_{2}$, see text), 2.12 (br m, 1H, $H^{5}$ ), 1.28-1.03 (m, 12H, $\left.\mathrm{P}^{1,2} \mathrm{CH}_{2} \mathrm{CH}_{3}\right) .{ }^{13} \mathrm{C}\left\{{ }^{1} \mathrm{H}\right\} \mathrm{NMR}: \delta 199.7\left(\mathrm{~s}, C^{1}\right), 163.3\left(\mathrm{~d},{ }^{3} J_{\mathrm{PC}}=7.9, C^{3}\right)$, $149.3\left(\mathrm{~d},{ }^{4} J_{\mathrm{PC}}=2.3, C^{13^{\prime}}\right), 148.9\left(\mathrm{~d},{ }^{4} J_{\mathrm{PC}}=3.0, C^{13}\right), 148.6\left(\mathrm{~s}, C^{9,9^{\prime}}\right), 140.4\left(\mathrm{~s}, C^{10}\right), 136.6$ $\left(\mathrm{d},{ }^{4} J_{\mathrm{PC}}=2.2, p-C\right.$ of $\left.\mathrm{P}^{1}\right), 136.5\left(\mathrm{~d},{ }^{4} J_{\mathrm{PC}}=2.3, p-C\right.$ of $\left.\mathrm{P}^{2}\right), 136.4\left(\mathrm{~d},{ }^{5} J_{\mathrm{PC}}=3.3, C^{14}\right), 134.2$ $\left(\mathrm{d},{ }^{3} J_{\mathrm{PC}}=8.2, m-C\right.$ of $\left.\mathrm{P}^{1}\right), 133.2\left(\mathrm{~d},{ }^{3} J_{\mathrm{PC}}=8.4, m-C\right.$ of $\left.\mathrm{P}^{2}\right), 131.8\left(\mathrm{~d},{ }^{2} J_{\mathrm{PC}}=11.8, o-C\right.$ of $\left.\mathrm{P}^{1}\right)$, $131.4\left(\mathrm{~d},{ }^{2} J_{\mathrm{PC}}=11.5, o-C\right.$ of $\left.\mathrm{P}^{2}\right), 127.4\left(\mathrm{~d},{ }^{2} J_{\mathrm{PC}}=6.1, C^{2}\right), 125.0\left(\mathrm{~d},{ }^{3} J_{\mathrm{PC}}=1.7, C^{7}\right), 120.0$ $\left(\mathrm{d},{ }^{2} J_{\mathrm{PC}}=6.3, C^{11}\right), 117.4\left(\mathrm{~d},{ }^{1} J_{\mathrm{PC}}=77.7, C_{\mathrm{ipso}}-\mathrm{P}^{1}\right), 116.1\left(\mathrm{~s}, C^{7}\right), 115.3\left(\mathrm{~d},{ }^{1} J_{\mathrm{PC}}=77.4\right.$, $\left.C_{\text {ipso }}-\mathrm{P}^{2}\right), 110.5\left(\mathrm{~d},{ }^{3} J_{\mathrm{PC}}=5.9, C^{12^{\prime}}\right), 109.3\left(\mathrm{~s}, C^{8,8^{\prime}}\right), 103.5\left(\mathrm{~d},{ }^{3} J_{\mathrm{PC}}=3.4, C^{12}\right), 57.4(\mathrm{~s}$, 


\section{Table S2 (cont).}

$\left.\mathrm{OCH}_{3}{ }^{9,9^{\prime}}\right), 57.1\left(\mathrm{~s}, \mathrm{OCH}_{3}{ }^{13}\right), 56.9\left(\mathrm{~s}, \mathrm{OCH}_{3}{ }^{13}\right), 38.9\left(\mathrm{dd},{ }^{1} J_{\mathrm{PC}}=44.2,{ }^{3} J_{\mathrm{PC}}=13.7, C^{6}\right), 34.5$ $\left(\mathrm{dd},{ }^{1} J_{\mathrm{PC}}=46.5,{ }^{3} J_{\mathrm{PC}}=14.3, C^{4}\right), 25.2\left(\mathrm{~s}, C^{5}\right), 13.5\left(\mathrm{~d},{ }^{1} J_{\mathrm{PC}}=47.8, \mathrm{P}^{1} C \mathrm{H}_{2}{ }^{\prime}\right), 12.5\left(\mathrm{~d},{ }^{1} J_{\mathrm{PC}}=\right.$ 45.8, $\left.\mathrm{P}^{1} \mathrm{CH}_{2}\right), 11.4\left(\mathrm{~d},{ }^{1} J_{\mathrm{PC}}=46.3, \mathrm{P}^{2} \mathrm{CH}_{2}\right), 10.6\left(\mathrm{~d},{ }^{1} J_{\mathrm{PC}}=51.3, \mathrm{P}^{2} \mathrm{CH}_{2}{ }^{\prime}\right), 7.2\left(\mathrm{~d},{ }^{2} J_{\mathrm{PC}}=5.6\right.$, $\left.\mathrm{P}^{1} \mathrm{CH}_{2} \mathrm{CH}_{3}{ }^{\prime}\right), 7.1\left(\mathrm{~d},{ }^{2} J_{\mathrm{PC}}=5.4, \mathrm{P}^{1} \mathrm{CH}_{2} C_{3}\right), 6.2\left(\mathrm{~d},{ }^{2} J_{\mathrm{PC}}=4.9, \mathrm{P}^{2} \mathrm{CH}_{2} C \mathrm{H}_{3}\right), 6.0\left(\mathrm{~d},{ }^{2} J_{\mathrm{PC}}=\right.$ 4.9, $\left.\mathrm{P}^{2} \mathrm{CH}_{2} \mathrm{CH}_{3}{ }^{\prime}\right)$. UV-Vis: 246, $362 \mathrm{~nm}$. Low-resolution ESI MS $\left(\mathrm{H}_{2} \mathrm{O}\right): m / 2 z 366.3$ (100\%), [7g $-2 \mathrm{Cl}]^{2+}$, calcd 366.7; m/z 565.2 (62\%), [7g - 2Cl- $\left.\mathrm{Et}_{2} \mathrm{PhP}-\mathrm{H}\right]^{+}$, calcd 565.2; $m / z 767.1(32 \%),[7 \mathrm{~g}-\mathrm{Cl}]^{+}$, calcd 767.3; m/z $183.0(20 \%),\left[\mathrm{Et}_{2} \mathrm{PhPO}+\mathrm{H}\right]^{+}$, calcd 183.1; $\left.m / z 167.0(20 \%),\left[\mathrm{Et}_{2} \mathrm{PhP}+\mathrm{H}\right]^{+}\right)$, calcd 167.1. 